\title{
Crowdsourcing inhibitor discovery against the SARS-CoV-2 main protease
}

\author{
N London ${ }^{1}$ \\ ${ }^{1}$ Weizmann Institute of Science \\ nir.london@weizmann.ac.il
}

COVID-19, caused by SARS-CoV-2, lacks effective therapeutics. Additionally, no antiviral drugs or vaccines were developed against the closely related coronavirus, SARS-CoV-1 or MERS-CoV, despite previous zoonotic outbreaks. To identify starting points for such therapeutics, we performed a large-scale screen of electrophile and non-covalent fragments through a combined mass spectrometry and X-ray approach against the SARS-CoV-2 main protease. The results with more than 71 co-crystal structures that span the entire active site, were released to the public and we implement a crowed-sourcing platform to solicit next generation design ideas. Thousands of suggestions were submitted, of which we made and tested hundreds, through a global consortium including academic labs, chemical vendors, pharma advisors and more. This effort resulted in sub-uM inhibitors with crystallographic confirmation that are now being further developed and assessed by live virus assays. We hope this initiative can serve as a template for an alternative drug discovery pipeline for areas that are unappealing to traditional pharma such as pandemic preparedness and antibiotic development.

Acta Cryst. (2020). A76, a229 